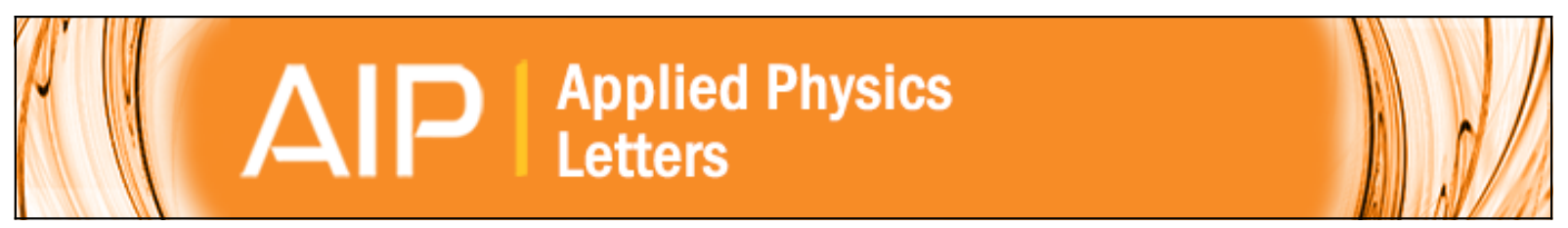

A micrometer-size movable light emitting area in a resonant tunneling light emitting diode

G. Pettinari, N. Balakrishnan, O. Makarovsky, R. P. Campion, A. Polimeni, M. Capizzi, and A. Patanè

Citation: Applied Physics Letters 103, 241105 (2013); doi: 10.1063/1.4844975

View online: http://dx.doi.org/10.1063/1.4844975

View Table of Contents: http://scitation.aip.org/content/aip/journal/apl/103/24?ver=pdfcov

Published by the AIP Publishing

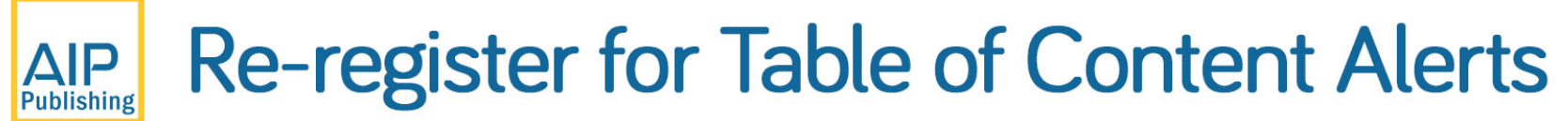




\title{
A micrometer-size movable light emitting area in a resonant tunneling light emitting diode
}

\author{
G. Pettinari, ${ }^{1,2, a)}$ N. Balakrishnan, ${ }^{1}$ O. Makarovsky, ${ }^{1}$ R. P. Campion, ${ }^{1}$ A. Polimeni, ${ }^{3}$ \\ M. Capizzi, ${ }^{3}$ and A. Patanè ${ }^{1}$ \\ ${ }^{1}$ School of Physics and Astronomy, University of Nottingham, Nottingham NG7 2RD, United Kingdom \\ ${ }^{2}$ National Research Council (CNR), Institute for Photonics and Nanotechnologies (IFN-CNR), \\ Via Cineto Romano 42, 00156 Roma, Italy \\ ${ }^{3}$ CNISM-Dipartimento di Fisica, Sapienza Università di Roma, P.le A. Moro 2, 00185 Roma, Italy
}

(Received 3 October 2013; accepted 22 November 2013; published online 10 December 2013)

\begin{abstract}
We report on the fabrication of a micrometer-size movable light emitting area in a GaAs/AlAs quantum well resonant tunneling $p-i-n$ diode. The spatial position of the micrometer-size light emitting area shifts linearly with increasing applied bias, up to $30 \mu \mathrm{m}$ for a bias increment of $0.2 \mathrm{~V}$. Also, the simultaneous resonant tunneling injection of both electrons and holes into the quantum well states is achieved at specific positions of the diode, thus resulting in a tenfold increase of the electroluminescence intensity. (C) 2013 AIP Publishing LLC. [http://dx.doi.org/10.1063/1.4844975]
\end{abstract}

The ability to spatially localize ${ }^{1-6}$ and move $\mathrm{e}^{7-9}$ on a micrometer scale the emitting region in a light emitting diode (LED) is relevant for several applications including LabOn-a-Chip experiments, bio-imaging, high-resolution micro-displays, optoelectronic integrated circuits, etc. A movable micrometer-size light emitting area has been achieved, recently, in organic LEDs (OLEDs) and transistors (OLETs). ${ }^{7-9}$ However, low quantum efficiencies and low carrier mobilities, typical of organic materials, often make necessary high applied voltages to generate spatially controlled light. ${ }^{7,9}$ Temporal instabilities, low control on spatial profiles, and light quenching have also been observed. ${ }^{8-10}$ These drawbacks could be overcome in an inorganic LED, where the spatial control on a micrometer scale of the light emitting area is therefore highly desirable. So far, micrometer- and nanometer-size light emitting areas have been created in inorganic LEDs by different approaches, including lithographic ${ }^{1-4}$ and laser-writing ${ }^{5,6}$ techniques. However, these approaches do not provide a means of moving spatially the light emitting area created.

In this Letter, we report on the realization of a biascontrolled, micrometer-size movable light emitting area in an inorganic resonant tunneling LED (RT-LED) containing a GaAs/AlAs quantum well (QW). Our approach exploits the electrical injection of charge carriers into the quantum states of the QW and the control of this process by post-growth hydrogen implantation. We show that the position of the QW electroluminescence (EL) area can be spatially shifted by the applied voltage and that the bias condition for the resonant injection of electrons and holes into the bound states of the QW changes across the mesa. In turn, this can lead to the $s i$ multaneous resonant tunneling injection of electrons and holes into the QW at specific positions of the diode, thus resulting in a tenfold increase of the local EL intensity. As several innovative device concepts in emerging nanotechnologies exploit the quantum tunneling of charge carriers into a quantum state, our approach could be extended to other applications of resonant tunneling that span from optoelectronic

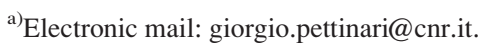

integrated circuits $^{11,12}$ to electrically pumped quantum devices. $^{13,14}$

Our GaAs/AlAs QW resonant tunneling structure was grown by molecular beam epitaxy on a (001) $n^{+}$-GaAs substrate and has the following layer layout, in order of growth: a $300 \mathrm{~nm}$ contact layer of $n^{+}$-GaAs (Si-doped to $2 \times 10^{18} \mathrm{~cm}^{-3}$ ), a $100 \mathrm{~nm}$ buffer layer of $n$-GaAs (Si-doped to $2 \times 10^{17} \mathrm{~cm}^{-3}$ ), an undoped central intrinsic region comprising a $20 \mathrm{~nm}$ GaAs spacer layer, a $5 \mathrm{~nm}$ AlAs tunnel barrier, a $6 \mathrm{~nm} \mathrm{GaAs}$ QW, a $5 \mathrm{~nm} \mathrm{AlAs} \mathrm{tunnel} \mathrm{barrier,} \mathrm{and} \mathrm{a} 10 \mathrm{~nm}$ GaAs spacer layer, and finally, a $50 \mathrm{~nm}$ contact layer of $p^{+}$-GaAs (C-doped to $1 \times 10^{19} \mathrm{~cm}^{-3}$ ); all layers were grown at $600{ }^{\circ} \mathrm{C}$. The epilayer was processed into $200 \mu \mathrm{m}$ and $400 \mu \mathrm{m}$ diameter mesa diodes with a top ring-shaped Ti/Au electrical contact for optical access. The processed devices were exposed to a low-energy $(100 \mathrm{eV}) \mathrm{H}^{+}$ion beam generated by a Kaufman source under different impinging doses (ranging from $6 \times 10^{16}$ ions $/ \mathrm{cm}^{2}$ to $1 \times 10^{19}$ ions $/ \mathrm{cm}^{2}$ ) and temperatures $T$ (ranging from $20^{\circ} \mathrm{C}$ to $250^{\circ} \mathrm{C}$ ). EL maps and spectra were acquired in a cold-finger optical cryostat using a confocal spectrometer equipped with a $50 \times$ objective (numerical aperture $=0.55$ ), a nano-focusing system, a XY scanning stage, a $0.5 \mathrm{~m}$ focal-length monochromator, and a liquid-nitrogen cooled Si charge coupled device (CCD). DC transport measurements were performed in a liquid-helium bath cryostat with a low-noise $(<0.1 \mathrm{pA})$ setup.

Figure 1 shows the realization of a bias-controlled movable light emitting area by post-growth hydrogenation of a RT-LED. The low temperature $(T=4.2 \mathrm{~K})$ current-voltage $I(V)$ characteristics of the untreated (virgin) device is shown in Fig. 1(a). The $I(V)$ curve is dominated by a strong resonance due to tunneling of electrons into the lowest conduction subband (E1) of the QW and by much weaker resonances associated with tunneling of holes into the heavyhole (HH1 and HH2) and light-hole (LH1) valence subbands of the QW, as observed before in similar RT-LEDs. ${ }^{15,16}$ Following hydrogen implantation on the mesa diode, the resistivity of the $p$-type GaAs layer increases, whereas the $I(V)$ curve is weakly affected and preserves its main resonant features. Resistance and capacitance measurements indicate that 
the $p$-type resistivity increases by increasing the implanted hydrogen dose and/or the hydrogenation temperature. In particular, for an hydrogen treatment at $T=250{ }^{\circ} \mathrm{C}$ with a total $\mathrm{H}$-impinging dose of $1 \times 10^{19}$ ions $/ \mathrm{cm}^{2}$, we observe a resistivity increase larger than four orders of magnitude. These hydrogenation conditions also lead to a pronounced change in the spatial profile of the QW EL intensity. Indeed, before hydrogen incorporation, the QW EL intensity is uniform across the mesa diode (Fig. 1(c)), while after hydrogenation it is localized along a closed line that hereafter we name "ring" (Fig. 1(d)). The light emitting area has a Lorentzian spatial profile with full-width at half-maximum $(w)$ of $3.3 \pm 0.1 \mu \mathrm{m}$ and moves linearly with increasing the applied voltage (Fig. 1(e)). For a movie of the bias-controlled movable RT-LED, see multimedia view of Fig. 1(d).

We now consider in detail the characteristics of the movable RT-LED. We found that: (i) the QW EL intensity is significantly larger (more than two orders of magnitude) on the ring than outside the ring (Fig. 2(a)) for each applied bias and position of the ring; (ii) the QW EL intensity on the ring is much stronger than that on the virgin sample and it is resonantly enhanced at a characteristic bias of
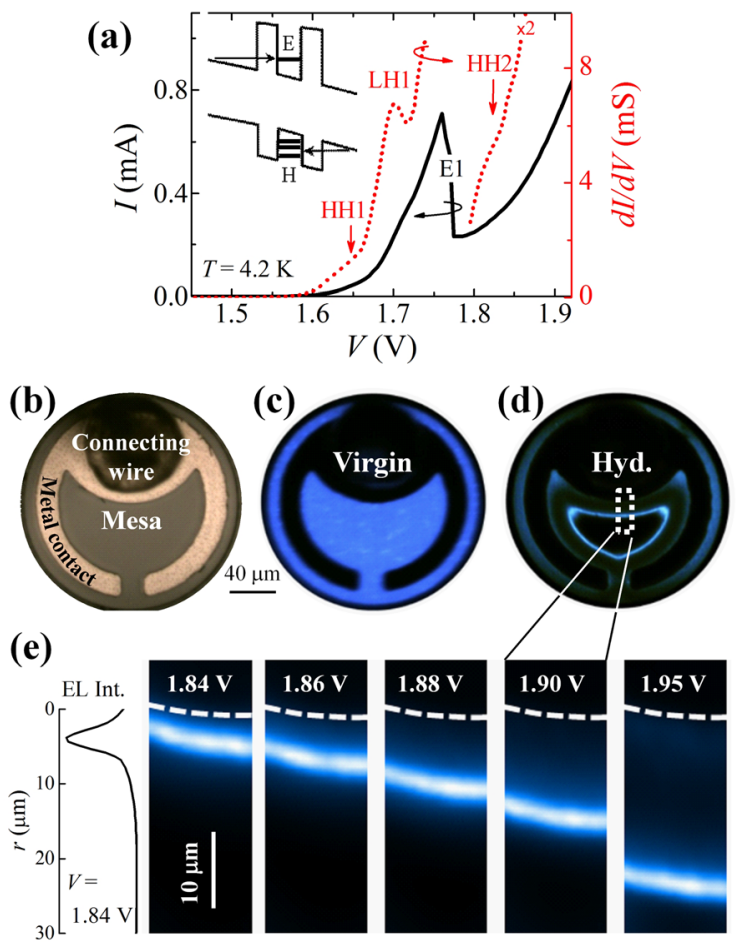

FIG. 1. A movable light emitting area by post-growth hydrogenation of a RT-LED. (a) Current-voltage $I(V)$ characteristics of a virgin $200 \mu$ m diameter mesa RT-LED at $T=4.2 \mathrm{~K}$. This is dominated by a strong resonance due to tunneling of electrons into the E1 QW subband and by much weaker resonances-observed in the conductance $d I / d V$ - due to tunneling of holes into the HH1, LH1, and HH2 QW subbands; see inset for a sketch of the carrier injection into the QW. (b) Optical image of a $200 \mu \mathrm{m}$ diameter mesa RT-LED. (c) and (d) Digital camera images of the EL emission intensity (at $T=9 \mathrm{~K}$ and $V=1.9 \mathrm{~V}$ ) before [Virgin, (c)] and after [Hyd., (d) (Multimedia view) [URL: http://dx.doi.org/10.1063/1.4844975.1]] post-growth hydrogen incorporation. The images were acquired with an $800 \mathrm{~nm}$ short-pass filter to remove the contribution from the GaAs spacer and contact layers. (e) Normalized EL maps for increasing values of the applied voltage, as obtained by plotting the EL intensity (at $T=9 \mathrm{~K}$ ) around the QW emission energy $(E=1.63 \mathrm{eV})$. The dashed lines in the EL maps indicate the metal contact edge. The EL spatial profile is also shown for $V=1.84 \mathrm{~V}$.
$V \sim 1.85 \mathrm{~V}$ (Fig. 2(b)); (iii) also, with increasing voltage, its position $r$-measured radially from the metal contact edge on the mesa-shifts linearly from the contact towards the center of the mesa at a rate of $0.142 \mu \mathrm{m} / \mathrm{mV}$, which corresponds to a shift of $\sim 30 \mu \mathrm{m}$ for a bias increment of $0.2 \mathrm{~V}$ (Fig. 2(c), see also multimedia view of Fig. 1(d)). Similar data were obtained in other mesa diodes.

The non-uniform spatial profile of the EL intensity in the hydrogenated RT-LEDs indicates that the injection of carriers into the QW is non-uniform across the mesa. To interpret this finding, we first note that hydrogen is implanted only in the regions of the diode that are not protected by the metal contact (see Fig. 1(b)). These are the regions providing optical access to the diode and where hydrogen tends to passivate the $\mathrm{C}$-dopants in the $p$-type GaAs contact layer by forming $\mathrm{C}-\mathrm{H}$ complexes ${ }^{17,18}$ (see also supplementary material, S1). ${ }^{19}$ The hydrogen-induced C-dopant passivation results into an increase of the resistivity in the hydrogenated area and causes a lateral voltage drop from the metal contact edge $(r=0)$ towards the center of the mesa. Thus, the resonant injection of electrons and holes into the QW subbands occurs at different positions for each applied bias (see Fig. 3(a); and also supplementary material, S2). ${ }^{19}$

To model the spatial dependence of the electron and hole injection into the QW, we consider the alignment of the Fermi energy in the $n$ - and $p$-type GaAs contact layers with the electron and hole QW subbands, respectively. The hydrogen-induced passivation of C-dopants on the $p$-type layer results in an increase of the depletion region width, which is wider in the center of the mesa than beneath the metal contact, where the $p$-type layer-not exposed to hydrogen-retains locally its pristine characteristic. Therefore, to account for the lateral voltage drop across the mesa, we

(a)

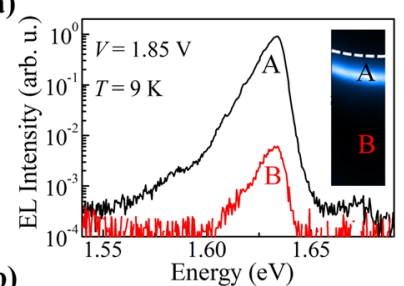

(b)

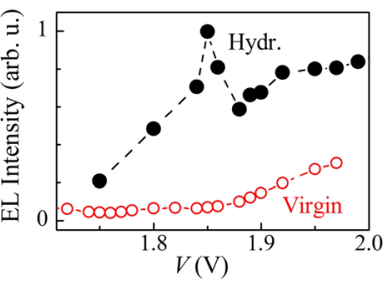

(c)

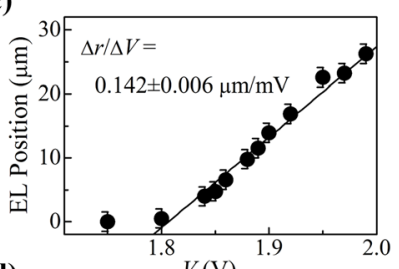

(d)

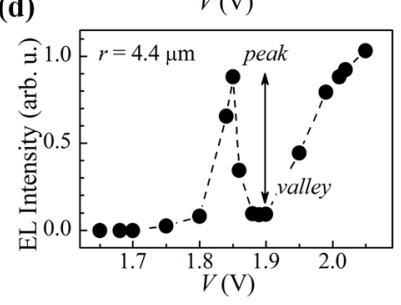

FIG. 2. Properties of the movable RT-LED. (a) QW EL spectrum on the ring (position $\mathrm{A}$ in the EL map) and outside the ring (position $\mathrm{B}$ in the EL map) at a given applied voltage $(V=1.85 \mathrm{~V})$. (b) Comparison of the bias dependence of the QW EL intensity in the hydrogenated sample (at the ring) and in the virgin sample (at the mesa center). In the virgin sample, the spatial inhomogeneity of the EL intensity is less than $4 \%$ and is independent on the applied voltage. (c) Position of the QW EL emission versus the applied voltage $V$ : the light emitting area shifts linearly from the contact edge towards the mesa center. The continuous line is a linear fit to the data at $V \geq 1.8 \mathrm{~V}$. (d) At a given position $(r=4.4 \mu \mathrm{m})$, the QW EL intensity versus the applied voltage shows a large peak-to-valley ratio $(\sim 10)$, much larger than the peak-to-valley ratio $(\sim 3)$ of the E1 peak in the current-voltage $I(V)$ characteristics (see Fig. 1(a)). 
(a)

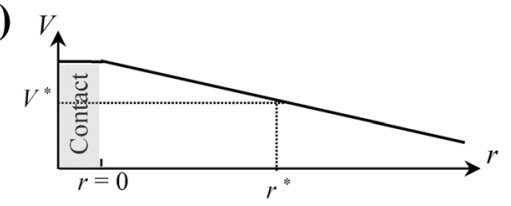

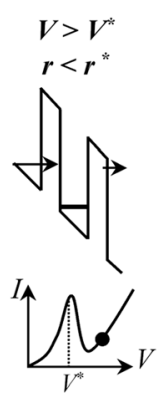

Off resonance

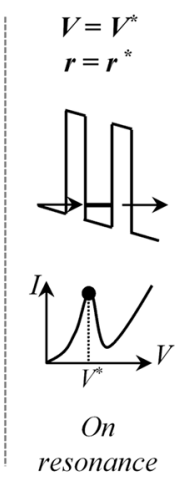

$V<V^{*}$

$r>r^{*}$
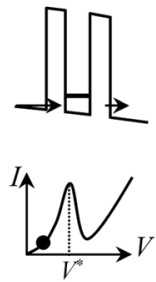

Off

resonance (b)

(c)

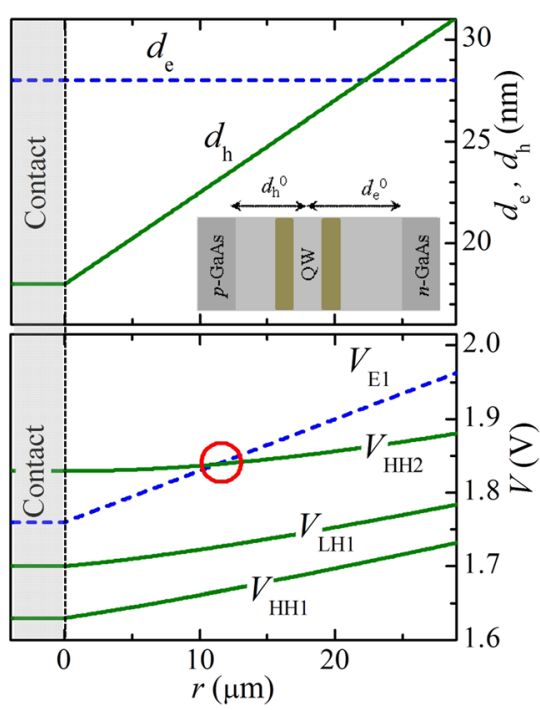

FIG. 3. Modeling of the movable RTLED. (a) Sketch of the spatial voltage drop along the mesa $-r$ being the radial distance from the metal contact edgeinduced by the hydrogen passivation of C-dopants in the $p$-type GaAs contact layer. This causes a spatial variation of the injection of carriers into the QW, with resonant injection occurring at a given position $r^{*}$. (b) Dependence on $r$ of the electron $\left(d_{\mathrm{e}}\right)$ and hole $\left(d_{\mathrm{h}}\right)$ tunneling distances and (c) of the tunneling voltages for the resonant injection of electrons $\left(V_{\mathrm{E} 1}\right)$ and holes $\left(V_{\mathrm{HH} 1}, V_{\mathrm{LH} 1}\right.$, and $V_{\mathrm{HH} 2}$ ) into the subbands of the QW. The simultaneous resonant injection of electrons and holes into the QW is highlighted by a red circle. consider a simple model that assumes a linear variation with $r$ of the hole tunneling distance on the $p$-type side of the structure (i.e., $d_{\mathrm{h}}=d_{\mathrm{h}}{ }^{0}+\alpha r$, where $\alpha$ is a constant) and a spatial independent electron tunneling distance on the $n$-type side (i.e., $d_{\mathrm{e}}=d_{\mathrm{e}}{ }^{0}$ ); see Fig. 3(b). The values of $d_{\mathrm{e}}$ and $d_{\mathrm{h}}$ at the contact edge, $d_{\mathrm{e}}{ }^{0}$ and $d_{\mathrm{h}}{ }^{0}$, are assumed equal, respectively, to the distances of the $n$ - and $p$-type GaAs layers from the center of the QW (see inset in Fig. 3(b)).

The spatial variation of $d_{\mathrm{e}}$ and $d_{\mathrm{h}}$ across the mesa results in a spatial change of the voltage, $\Delta V_{\mathrm{e}}$ and $\Delta V_{\mathrm{h}}$, dropped between the $n$ - and $p$-type GaAs layers and the center of the $\mathrm{QW}$, respectively. This is described by the electrostatic leverage factors for the conduction $\left[f_{\mathrm{e}}=\Delta V_{\mathrm{e}} / V=d_{\mathrm{e}} /\left(d_{\mathrm{e}}+d_{\mathrm{h}}\right)\right]$ and valence $\left[f_{\mathrm{h}}=\Delta V_{\mathrm{h}} / V=d_{\mathrm{h}} /\left(d_{\mathrm{e}}+d_{\mathrm{h}}\right)\right]$ bands. Using $f_{\mathrm{e}}$ and $f_{\mathrm{h}}$, we can write simple expressions for the voltage required for the resonant injection of electrons $\left[V_{\mathrm{E} 1}=V_{\mathrm{FB}}{ }^{0}+\left(V_{\mathrm{E} 1}{ }^{0}-V_{\mathrm{FB}}{ }^{0}\right) f_{\mathrm{e}}^{0}\right.$ $\left./ f_{\mathrm{e}}+\beta r\right]$ and holes $\left[V_{\mathrm{H}}=V_{\mathrm{FB}}{ }^{0}+\left(V_{\mathrm{H}}{ }^{0}-V_{\mathrm{FB}}{ }^{0}\right) f_{\mathrm{h}}{ }^{0} / f_{\mathrm{h}}+\beta r\right]$ into the E1 and hole $(\mathrm{H}=\mathrm{HH} 1, \mathrm{LH} 1, \mathrm{HH} 2)$ subbands of the QW, respectively. Here, $V_{\mathrm{FB}}{ }^{0}=1.51 \mathrm{~V}$ is the bias required to reach flat bands at $r=0$ and $\beta$ is a constant accounting for the linear voltage drop across the mesa caused by the spread resistance on the hydrogenated $p$-type contact layer.

As shown in Fig. 3(c), for each applied bias $V$, the resonant tunneling of electrons and holes into the QW subbands occurs at different positions $r$. In particular, the voltage for tunneling of holes into the $\mathrm{HH} 2$ subband $\left(V_{\mathrm{HH} 2}\right)$ crosses the voltage for electron tunneling into the E1 subband $\left(V_{\mathrm{E} 1}\right)$; see red circle in the figure. The crossing point corresponds to the simultaneous resonant injection of electrons and holes in the QW. By using $\alpha=0.45 \mathrm{~nm} / \mu \mathrm{m}$ and $\beta=4.6 \mathrm{mV} / \mu \mathrm{m}$, the model indicates that an applied voltage $V \sim 1.84 \mathrm{~V}$ is required to achieve simultaneous electron and hole resonant injection into the QW, thus accounting for the measured resonant enhancement of the QW EL intensity at $V=1.85 \mathrm{~V}$ (see Fig. 2(b)). ${ }^{20}$ Moreover, as the simultaneous resonant injection of carriers into the QW is satisfied under more stringent conditions than single charge resonant tunneling, the QW EL intensity versus the applied voltage is characterized by a peak-to-valley ratio $(\sim 10$, see Fig. $2(d))$ significantly larger than that $(\sim 3)$ observed for resonant tunneling of individual carriers (i.e., either electrons or holes). It is also larger than the peak-to-valley ratio ( 3 , see Fig. 1(a)) of the E1 resonance in $I(V)$. The model also indicates that the measured linear spatial shift of the EL emission with the bias is dominated by the electron injection; i.e., it follows the calculated $r$-dependence of $V_{\mathrm{E} 1}, \quad\left[d V_{\mathrm{E} 1}(r) / d r\right]^{-1}=\left[\beta+\alpha\left(V_{\mathrm{E} 1}{ }^{0}-V_{\mathrm{FB}}{ }^{0}\right)\right.$ $\left./\left(d_{\mathrm{e}}{ }^{0}+d_{\mathrm{h}}{ }^{0}\right)\right]^{-1}=0.142 \mu \mathrm{m} / \mathrm{mV}$.

The effects described above are observed for temperatures up to $T=100 \mathrm{~K}$. With increasing $T$, the ring becomes gradually less bright and broadens; see supplementary material, S3. ${ }^{19}$ As the temperature stability of the ring formation relies on the robustness of the resonant injection of carriers into the QW and on carrier thermal escape mechanisms, we envisage that optimization of the layer layout and/or the employment of a multi-barrier structure could make possible room temperature operation. ${ }^{21,22}$

In conclusion, we have reported on the fabrication of a micrometer-size movable light emitting area in a solid state GaAs/AlAs QW-based RT-LED. We have shown that the position of the light emitting area can be spatially shifted over tens of $\mu \mathrm{m}$ by varying the applied voltage by a few hundreds of $\mathrm{mV}$. We have also shown that the simultaneous resonant injection of electrons and holes into the QW subbands increases the EL intensity (by $>10$ times) and the peak-tovalley EL ratio (by $\sim 3$ times). The condition for the spatial control of the emitted light and for the simultaneous resonant carrier injection has been achieved by post-growth hydrogen passivation of C-dopants on the $p$-type contact layer of the diode. This technique could be implemented in other fields of research that use resonant tunneling devices for a variety of applications spanning from $\mathrm{THz}$ resonators ${ }^{23}$ to high efficiency single-photon detectors ${ }^{24}$ and room-temperature negative-differential-resistance devices. ${ }^{21,22}$

This work was supported by the EU (under Grant Agreement No. PIEF-GA-2010-272612), The Royal Society (RG110416), The University of Nottingham, and the Italian MIUR (under the FIRB project DeLIGHTeD, Prot. RBFR12RS1W). We thank the EPSRC National Center for III-V Technologies (K. Kennedy and R. Airey) for device processing and A. Nasir (The University of Nottingham) for assistance during sample preparation. 
${ }^{1}$ V. Poher, H. X. Zhang, G. T. Kennedy, C. Griffin, S. Oddos, E. Gu, D. S. Elson, J. M. Girkin, P. M. W. French, M. D. Dawson, and M. A. A. Neil, Opt. Express 15, 11196 (2007).

${ }^{2}$ V. Poher, N. Grossman, G. T. Kennedy, K. Nikolic, H. X. Zhang, Z. Gong, E. M. Drakakis, E. Gu, M. D. Dawson, P. M. W. French, P. Degenaar, and M. A. A. Neil, J. Phys. D: Appl. Phys. 41, 094014 (2008).

${ }^{3}$ M. Vitzethuma, R. Schmidta, P. Kiesela, P. Schafmeisterb, D. Reuterb, A. D. Wieckb, and G. H. Dohlera, Physica E 13, 143 (2002).

${ }^{4}$ S. Li and A. Waag, J. Appl. Phys. 111, 071101 (2012).

${ }^{5}$ O. Makarovsky, S. Kumar, A. Rastelli, A. Patanè, L. Eaves, A. G. Balanov, O. G. Schmidt, R. Campion, and C. T. Foxon, Adv. Mater. 22, 3176 (2010).

${ }^{6}$ F. Intonti, V. Matarazzo, A. Nasir, O. Makarovsky, R. Campion, A. Patanè, S. Kumar, A. Rastelli, O. G. Schmidt, and M. Gurioli, Appl. Phys. Lett. 98, 183102 (2011).

${ }^{7}$ J. Zaumseil, R. H. Friend, and H. Sirringhaus, Nature Mater. 5, 69 (2006).

${ }^{8}$ J. Liu, I. Engquist, X. Crispin, and M. Berggren, J. Am. Chem. Soc. 134, 901 (2012).

${ }^{9}$ B. B. Y. Hsu, C. Duan, E. B. Namdas, A. Gutacker, J. D. Yuen, F. Huang, Y. Cao, G. C. Bazan, I. D. W. Samuel, and A. J. Heeger, Adv. Mater. 24, 1171 (2012).

${ }^{10}$ S. W. Culligan, A. C.-A. Chen, J. U. Wallace, K. P. Klubek, C. W. Tang, and S. H. Chen, Adv. Funct. Mater. 16, 1481 (2006).

${ }^{11}$ F. Capasso, K. Mohammed, and A. Y. Cho, IEEE J. Quantum Electron. 22, 1853 (1986).

${ }^{12} \mathrm{H}$. Mizuta and T. Tanoue, The Physics and Applications of Resonant Tunnelling Diodes (Cambridge University Press, UK, 1995).

${ }^{13}$ J. Kim, O. Benson, H. Kan, and Y. Yamamoto, Nature 397, 500 (1999).
${ }^{14}$ A. J. Shields, Nature Photon. 1, 215 (2007).

${ }^{15}$ P. M. Martin, R. K. Hayden, C. R. H. White, M. Henini, L. Eaves, D. K. Maude, J. C. Portal, G. Hill, and M. A. Pate, Semicond. Sci. Technol. 7, B456 (1992).

${ }^{16}$ C. R. H. White, H. B. Evans, L. Eaves, P. M. Martin, M. Henini, G. Hill, and M. A. Pate, Phys. Rev. B 45, 9513 (1992).

${ }^{17}$ N. Pan, S. S. Bose, M. H. Kim, G. E. Stillman, F. Chambers, G. Devane, C. R. Ito, and M. Feng, Appl. Phys. Lett. 51, 596 (1987).

${ }^{18}$ R. Rahbi, B. Pajot, J. Chevallier, A. Marbeuf, R. C. Logan, and M. Gavand, J. Appl. Phys. 73, 1723 (1993).

${ }^{19}$ See supplementary material at http://dx.doi.org/10.1063/1.4844975 for optical identification of $\mathrm{H}$ incorporation, simulations of the EL spatial localization, and temperature studies.

${ }^{20}$ The calculated position at which the simultaneous resonant injection occurs $(r=11.5 \mu \mathrm{m})$ differs from the experimental value $(r=4.4 \mu \mathrm{m})$ by an amount that corresponds to the extrapolated value of the ring position $(r=-6.6 \mu \mathrm{m}$, see Fig. $2(\mathrm{c}))$ at the E1 tunneling voltage $\left(V_{\mathrm{E} 1}{ }^{0}=1.76 \mathrm{~V}\right)$. This offset is likely to originate from an additional contact voltage drop, from the experimental uncertainty in defining the contact edge position from the EL maps, and/or from the hydrogen diffusion beneath the contact, all factors which have not been included in the model.

${ }^{21}$ C. Bayram, Z. Vashaei, and M. Razeghi, Appl. Phys. Lett. 97, 092104 (2010).

${ }^{22}$ Y. Suda and H. Koyama, Appl. Phys. Lett. 79, 2273 (2001).

${ }^{23}$ M. Feiginov, C. Sydlo, O. Cojocari, and P. Meissner, Appl. Phys. Lett. 99, 233506 (2011).

${ }^{24}$ J. C. Blakesley, P. See, A. J. Shields, B. E. Kardynał, P. Atkinson, I. Farrer, and D. A. Ritchie, Phys. Rev. Lett. 94, 067401 (2005). 